\title{
Integer Optimization Methods for Non-MSE Data Compression for Emitter Location
}

\author{
Mark L. Fowler ${ }^{\dagger}$ and Mo Chen \\ Department of Electrical and Computer Engineering \\ State University of New York at Binghamton \\ Binghamton, NY 13902
}

\begin{abstract}
The location of an emitter is estimated by intercepting its signal and sharing the data among several platforms to measure the time-difference-of-arrival (TDOA) and the frequency-difference-of-arrival (FDOA). Doing this in a timely fashion requires effective data compression. A common compression approach is to use a rate-distortion criterion where distortion is taken to be the mean-square error (MSE) between the original and compressed versions of the signal. However, in this paper we show that this MSE-only approach is inappropriate for TDOA/FDOA estimation and then define a more appropriate, nonMSE distortion measure. This measure is based on the fact that in addition to the dependence on MSE, the TDOA accuracy also depends inversely on the signal's RMS (or Gabor) bandwidth and the FDOA accuracy also depends inversely on the signal's RMS (or Gabor) duration.

We develop specific forms of this new non-MSE distortion measure and apply it to the case of using the DFT for compression. The form of this new measure must be optimized under the constraint of a specified budget on the total number of bits available for coding. We show that this optimization requires a selection of DFT cells to retain that must be jointly chosen with an appropriate allocation of bits to the selected DFT cells. This joint selection/allocation is a challenging integer optimization problem that still has not been solved. However, we consider three possible sub-optimal approaches and compare their performance.
\end{abstract}

Keywords: data compression, emitter location, time-difference-of-arrival (TDOA), frequency-difference-of-arrival (FDOA), wavelet transform, RMS bandwidth, Gabor bandwidth, dynamic programming, integer programming.

\section{INTRODUCTION}

A common way to locate electromagnetic emitters is to measure the time-difference-of-arrival (TDOA) and the frequency-difference-of-arrival (FDOA) between pairs of signals received at geographically separated sites ${ }^{1,2,3}$. The measurement of TDOA/FDOA between these signals is done by coherently cross-correlating the signal pairs, ${ }^{2,3}$ and requires that the signal samples of the two signals are available at a common site, which is generally accomplished by transferring the signal samples over a data link from one site to the other. An important aspect of this that is not widely addressed in the literature is that often the available data link rate is insufficient to accomplish the transfer within the time requirement unless some form of lossy data compression is employed. For the case of white Gaussian signals and noises, Matthiesen and Miller ${ }^{4}$ established bounds on the rate-distortion performance for the TDOA problem and compared them to the performance achievable using scalar quantizers, where distortion is measured in terms of lost SNR due to the mean square error (MSE) of lossy compression. However, these results are not applicable when locating radar and communication emitters because the signals encountered are not Gaussian. A method using block adaptive scalar quantization was proposed ${ }^{5}$ and analyzed $^{6}$ to show that it was marginally effective for various signal types. Wavelet-based methods have been proposed ${ }^{7}$ and demonstrated $^{8}$ to give compression ratios on the order of 4 to 7 for some radar signals. A method that optimally trades between decimation and quantization has been developed for flat spectrum signals and shown to perform better than either method alone. ${ }^{9}$ A non-MSE distortion measure has been developed and exploited to improve wavelet-based compression; $;^{10,11}$ however, the method used to optimize the non-MSE distortion measure has not been thoroughly developed. We develop and compare three methods that exploit linear programming methods as a means to optimize the non-MSE distortion measure. For completeness of the presentation here, we repeat some supporting material given previously ${ }^{11}$.

The two signals to be correlated are the complex envelopes of the received RF signals. The two noisy received signals to be processed are notated as

\footnotetext{
† Correspondence: mfowler@binghamton.edu
} 


$$
\begin{gathered}
\hat{s}(k)=s(k)+n(k) \\
\hat{d}(k)=d(k)+v(k)
\end{gathered}
$$

where $s(k)$ and $d(k)$ are the complex baseband signals of interest and $n(k)$ and $v(k)$ are complex white Gaussian noises. The signal $d(k)$ is a delayed and doppler shifted version of $s(k)$. The signal-to-noise ratios (SNR) for these two signals are denoted $S N R$ and $D N R$, respectively. To cross correlate these two signals one of them (assumed to be $\hat{s}(k)$ here) is compressed, transferred to the other platform, and then decompressed before cross-correlation, as shown in Figure 1.

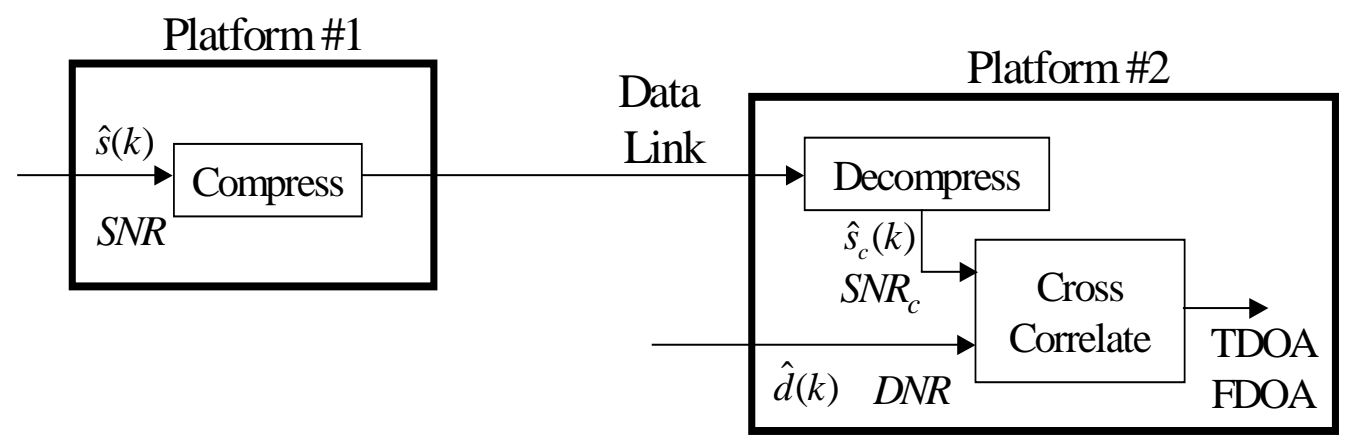

\section{Figure 1: System Configuration for Compression}

Signal $\hat{s}(k)$ has SNR of $S N R_{c}<S N R$ after lossy compression/decompression, and the output SNR after crosscorrelation is given by

$$
\begin{aligned}
S N R_{o} & =\frac{W T}{\frac{1}{S N R_{c}}+\frac{1}{D N R}+\frac{1}{S N R_{c} D N R}} \\
& \triangleq W T \times S N R_{\text {eff }}
\end{aligned}
$$

where $W T$ is the time-bandwidth product (or coherent processing gain), with $W$ being the noise bandwidth of the receiver and $T$ being the duration of the received signal and $S N R_{\text {eff }}$ is a so-called effective $\mathrm{SNR}^{3}$. From (2) it is clear that the correlator's output SNR is set by the lower of $S N R_{c}$ and $D N R$ : when one is smaller than the other we have

$$
S N R_{o} \approx W T \times \min \left\{S N R_{c}, D N R\right\} .
$$
$\mathrm{by}^{3}$

The accuracies of the TDOA/FDOA estimates are governed by the Cramer-Rao bound (CRB) for TDOA/FDOA given

$$
\begin{aligned}
\sigma_{T D O A} & \geq \frac{1}{2 \pi B_{r m s} \sqrt{2 S N R_{O}}} \\
\sigma_{F D O A} & \geq \frac{1}{2 \pi D_{r m s} \sqrt{2 S N R_{O}}}
\end{aligned}
$$

\footnotetext{
${ }^{\ddagger} \mathrm{SNR}$ (non-italic) represents an acronym for signal-to-noise ratio; SNR (italic) is a symbol representing the SNR for $\hat{s}(k)$.
} 
where $B_{r m s}$ is the signal's RMS (or Gabor) bandwidth in $\mathrm{Hz}$ given by

$$
B_{r m s}^{2}=\frac{\int f^{2}|S(f)|^{2} d f}{\int|S(f)|^{2} d f},
$$

with $S(f)$ being the Fourier transform of the signal $s(k)$ and $D_{r m s}$ is the signal's RMS duration in seconds given by

$$
D_{r m s}^{2}=\frac{\int t^{2}|s(t)|^{2} d t}{\int|s(t)|^{2} d t} .
$$

The denominators in (4) and (5) can be considered as normalizing factors on $|S(f)|^{2}$ and $|s(t)|^{2}$, respectively, so that these equations have the form identical to the equation for variance; thus, the root-mean-squared (RMS) terminology.

\section{NON-MSE DISTORTION CRITERIA}

In TDOA/FDOA applications it is crucial that the compression methods minimize the impact on the TDOA/FDOA estimation performance rather than stressing minimization of MSE as is common in many compression techniques. We have argued $^{11}$ using the forms of the CRB that we should take as measures to maximize for a given desired rate the following weighted "RMS-distortion" SNRs:

$$
\begin{aligned}
S N R_{r m s, T D O A} & =B_{r m s}^{2} S N R_{O} \\
& =B_{r m s}^{2}\left(W T \times S N R_{e f f}\right)
\end{aligned}
$$

and

$$
\begin{aligned}
S N R_{r m s, F D O A} & =D_{r m s}^{2} S N R_{O} \\
& =D_{r m s}^{2}\left(W T \times S N R_{e f f}\right)
\end{aligned} .
$$

The specific forms of these distortion measures depend on if $S N R>D N R$ or $S N R<D N R$. We consider here only the latter case, where we have shown ${ }^{11}$ that we can use effective RMS-distortion SNRs given by

$$
S N R_{T D O A}=\frac{\int f^{2}|S(f)|^{2} d f}{N_{o} W}
$$

and

$$
S N R_{F D O A}=\frac{\int t^{2}|s(t)|^{2} d t}{N_{o} W},
$$

where $N_{o}$ is the noise power spectral density level of the white noise.

So the general goal of our compression method is the following, expressed as transform coding ${ }^{12,13}$ with a non-MSE distortion. Given some orthogonal signal decomposition (e.g., wavelet transform, DFT, etc.)

$$
\hat{s}(k)=\sum_{n=1}^{N} c_{n} \psi_{n}(k)
$$


of the signal to be compressed, we wish to select which coefficients $c_{n}$ should be coded and transmitted to achieve a desired rate-distortion goal where distortion is measured using (8) and (9). Loosely stated, we wish to find a subset $\tilde{\Omega}$ of coefficient indices such that the signal given by

$$
\tilde{s}(k)=\sum_{n \in \tilde{\Omega}} c_{n} \psi_{n}(k)
$$

maximizes (8) and (9) while the set $\left\{c_{n} \mid n \in \tilde{\Omega}\right\}$ can be coded using the available rate $R$. In practice, in order to reduce the computational complexity of determining which coefficients to remove, the coefficients can be grouped into blocks of coefficients and then a block is either kept or discarded in its entirety.

In general, determining this selection and coding is quite difficult because of (i) the nonlinear, nonmonotonic relationship between the coefficients and the RMS widths, and (ii) the fact that removing a coefficient from $\tilde{\Omega}$ effects the numerator and denominator of (8) and (9). Furthermore, the simultaneous maximization of (8) and (9) can be difficult, especially given that there may be a different acceptable level of degradation on TDOA than there is on FDOA; this multi-objective issue is not considered here. We consider only the task of estimating TDOA and therefore only consider the task of compression to maximize (8). Previously ${ }^{11}$, these considerations led to a suboptimal algorithm based on removing wavelet coefficients that correspond to components that have little impact on the numerator of (8). This eliminates coefficients that are insignificant on the basis of RMS bandwidth. Then bits are allocated to the remaining groups on the basis of the unweighted coefficients in order to achieve a desired MSE distortion; this step attempts to minimize the increase in the denominator of (8) due to the quantization.

\section{RMS SNR MAXIMIZATION METHODS}

What we consider here are three alternative methods to attack the maximization of (8) - none of them is optimal, however. We also consider only the use of the DFT as the transform; the DFT is chosen not because it is necessarily the best transform to use, but because it is simple to apply and our focus here is the relative performance of the optimization methods used to maximize (8) under the constraint of a total number of bits $R$.

In the absence of compression, for the $N$-point DFT $S[k], k=-N / 2,-N / 2+1, \ldots, N / 2-1$ having frequency bin size $\Delta f$, the measure in (8) becomes

$$
S N R_{T D O A}=\frac{\sum_{k=-N / 2}^{N / 2-1}(k \Delta f)^{2}|S[k]|^{2}}{N_{0} \Delta f N}
$$

Let the set of integers $\Omega=\{-N / 2,-N / 2+1, \ldots, N / 2-1\}$ be the set of indices of the DFT coefficients. Let the selection set $\tilde{\Omega} \subseteq \Omega$ be the subset of indices of the DFT coefficients that are kept during compression, let $B=\left\{b_{i} \mid i \in \tilde{\Omega}\right\}$ be the set of bit allocations for the selection set (i.e., the $i^{\text {th }}$ DFT coefficient in $\tilde{\Omega}$ is allocated $b_{i}$ bits for quantization), and let $P_{q}(B)$ be the quantization noise power due to this allocation. Then (12) becomes

$$
\begin{aligned}
\operatorname{SNR}_{T D O A}(\tilde{\Omega}, B) & =\frac{\sum_{k \in \tilde{\Omega}}(k \Delta f)^{2}|S[k+N / 2]|^{2}}{\left[N_{0} \sum_{k \in \tilde{\Omega}} \Delta f\right]+P_{q}(B)} \\
& =\frac{\sum_{k \in \tilde{\Omega}}(k \Delta f)^{2}|S[k+N / 2]|^{2}}{N_{0} \Delta f|\tilde{\Omega}|+P_{q}(B)}
\end{aligned}
$$

where $|\tilde{\Omega}|$ denotes the number of elements in the selection set $\tilde{\Omega}$. Note that the numerator of (13) is impacted only by the coefficient selection while the denominator is impacted by both the coefficient selection and the subsequent bit allocation. 
Note also that eliminating DFT coefficients acts to reduce the denominator (which acts to improve $S N R_{T D O A}$ ) while quantizing the remaining DFT coefficients acts to increase the denominator (which acts to degrade $S N R_{T D O A}$ ).

In the following algorithms we use the noisy DFT $\hat{S}[k]$ in (13) because that is what we can measure and we assume that some adjunct processing has provided us with an estimate of the white noise power spectral density level $N_{o}$. Also, all algorithms will be based on DFT coefficients being grouped into blocks of $M$ coefficients each: (i) an allocation of $b_{i}$ bits to the $i^{\text {th }}$ block causes all coefficients in the block to be quantized using $b_{i} / M$ bits, and (ii) discarding the $i^{\text {th }}$ block causes all coefficients in that block to be discarded.

\section{Select-Then-Allocate (STA)}

From the above we can state the true optimization problem that we wish to solve: jointly determine the selection set $\tilde{\Omega}$ and allocation $B$ in order to maximize (13). The joint selection-allocation problem is quite challenging to solve; therefore, we develop an alternative here. We wish to optimally select first and then optimally allocate.

It is straight-forward to use integer programming to find, for a given integer $\eta$, the best set of $\eta$ blocks of DFT coefficients to remove in order to maximize the numerator of (13). Once that is complete it is again straight-forward to use dynamic programming to allocate bits to the DFT coefficients remaining after the first step. But, how do we know the optimal number of blocks to remove? This is handled here iteratively:

1. Initialize to $\eta=1$

2. Select: Use integer programming to select $\widetilde{\Omega}$ by removing $\eta$ blocks to maximize the numerator of (13).

3. Allocate: Use dynamic programming to allocate the budgeted $R$ bits to the selected blocks.

4. Measure: Compute (13) for this selection/allocation to give $S N R_{T D O A}(\eta)$, where the change in argument for (13) should be obvious.

5. Increment $\eta$ by one and go to Step \#2. Repeat until a specified maximum number of blocks have been removed.

6. $\quad$ Assess: Choose the selection/allocation that gives the largest $S N R_{T D O A}(\eta)$.

While this method comes close to a direct attack on the true optimization problem, it is computationally complex. However, it serves as a measure to which other, less complex methods can be compared.

\section{Allocate-Then-Select (ATS)}

This method formulates an approach to attack the maximization of (13) by casting it into a form that is identical to the so-called knapsack problem (a well-known problem in integer programming ${ }^{14}$ ). Although this is not as direct an attack as in the Select-Then-Allocate Method, it has the advantage that the computational complexity is much less.

The knapsack problem is: given a set $\Lambda$ of objects with the $i^{\text {th }}$ element having a cost of $c_{i}$ and a utility of $u_{i}$, select the set of objects $\tilde{\Lambda} \subseteq \Lambda$ that maximizes the total utility $U$ given by

$$
U=\sum_{i \in \tilde{\Lambda}} u_{i}
$$

while ensuring that the total cost $C$ remains below some specified cost budget $C_{B}$, that is

$$
C=\sum_{i \in \tilde{\Lambda}} c_{i} \leq C_{B} .
$$

Well-known integer programming methods exist to solve the knapsack problem ${ }^{14}$.

The application of the knapsack problem to maximizing (13) is as follows. The user specifies a desired SQR (signal-toquantization power ratio); the method then determines the proper choice of quantizer cell size according to

$$
\Delta=\sqrt{\frac{12 P_{x}}{S Q R}}
$$

and assigns just enough bits to each block to allow all coefficients in the block to be covered by the quantizer; this allocation has been shown to achieve the desired SQR when using an orthogonal transform ${ }^{7}$. It is likely that the number of bits allocated using this scheme will exceed the budgeted total number of bits $R$. Thus, we now have a formulation that matches the knapsack problem. The cost of the $i^{\text {th }}$ block is given by the number of bits $b_{i}$ allocated to it, whereas its utility is measured by its contribution to the numerator of (13) given by 


$$
u_{i}=\sum_{k \in\left(i^{\mathrm{th}} \mathrm{Block}\right)}(k \Delta f)^{2}|S[k]|^{2}
$$

Integer programming is then used to select the blocks that will maximize the total utility while ensuring that the total number of bits allocated does not exceed the budgeted total number of bits $R$. Clearly maximizing the total utility is equivalent to maximizing the numerator in (13) and the first step of allocating bits to achieve a desired SQR is somewhat related to minimizing the quantization power $P_{q}$ in the denominator of (13). However, it is also clear that this approach is not an optimal approach to maximizing (13) as a whole. Nonetheless, it is a useful and interesting approach.

\section{Allocation Forced Selection (AFS)}

In standard transform-based or subband-coding compression schemes, bits are allocated to minimize some MSE-based distortion measure; but, when the signal is audio, for example, it is desirable to allocate bits based on some perceptual criterion $^{12,13}$. Although not exact, an effective, practical way to incorporate perceptual characteristics into the bit allocation process is to use a perceptually-weighted MSE criterion ${ }^{13}$. This idea is the basis for the method described in this subsection, although it is not "perception" that is used as a weighting factor but rather the insight given by the numerator of (13) is used.

In particular, standard MSE-based bit allocations when using the DFT would allocate bits according to the squared magnitude of the DFT - allocating more bits to DFT coefficients with larger squared magnitude. Here, however, motivated by the view that the numerator of (13) implies that high-frequency components are "quadratically more important" we use this as a low-complexity approach. The basic idea of the approach is to use dynamic programming to allocate $R$ bits to the DFT coefficient blocks in order to minimize

$$
\sum_{k=-N / 2}^{N / 2-1}(k \Delta f)^{2}\left|S[k]-S_{q}[k]\right|^{2}
$$

where $S_{q}[k]$ is the quantized DFT coefficients. Clearly, compared to the MSE-based approach this tends to allocate more bits to the higher frequency DFT coefficients since quantization errors there are penalized quadratically higher than errors at low frequencies. Also, when the total bit budget $R$ is small enough to require it (usually the case), this will have the effect of allocating zero bits to some DFT coefficients - thus, effectively removing them; therefore, this method uses the weightdriven allocation to force selection of DFT coefficients that are more important to TDOA estimation - hence we call this the "Allocation Forced Selection" method.

Although the methods discussed here are general and applicable to all varieties of signals we demonstrate the results here on a radar signal. The standard deviation of the TDOA estimation error is plotted versus the compressed signal's SNR for the case of DNR $=40 \mathrm{~dB}$. From this plot we see the relative performance of the various select/allocate methods described here. We see that the classical MSE approach performs worse than all of the non-MSE methods developed here - as expected. This verifies the validity of the non-MSE approach. Furthermore, we see that the selection/allocation method that most directly attacks the maximization of our proposed criterion in (13), namely the STA method, outperforms the other methods. We also see that the STA and AFS methods are comparable - it should be noted that AFS is considerably less complex than STA.

\section{CONCLUSIONS}

Compression algorithms intended for TDOA/FDOA applications should be developed using a distortion measure that focuses on the TDOA/FDOA accuracy not solely on MSE. We showed that it is possible to use the Cramer-Rao bound to develop a non-MSE distortion measure that captures this requirement. However, the form that this measure takes on leads to an objective function that poses a very challenging and difficult optimization problem. To explore methods of attacking this optimization problem we focused only the use of the DFT as the orthogonal transform used in the compression method - this allowed us to focus on the differences between the optimization approaches. It should be noted that use of a more suitable orthogonal transform (e.g., the wavelet transform) should lead to better compression results. However, the results presented here clearly demonstrate the usefulness of our non-MSE viewpoint as well as some of the methods we have developed to attack the optimization of the non-MSE distortion measure.

The optimization of the non-MSE distortion measure requires a subset of transform coefficients to be jointly selected and coded (where the coding requires the allocation of bits to the selected coefficients). However, the selection and allocation tasks were seen to be coupled in a way that makes this joint optimization difficult. We have used integer and dynamic programming in three different ways to accomplish our goals here. The methods differ mostly in the order of performing the tasks of selection and allocation: one method first selected and then allocated, one method first over-allocated and then 
selected to meet the bit budget, and a third simply allocated in a way that forced the selection through allocation of zero bits to coefficients deemed less important. It was found that the method that first selected and then allocated provides the best performance and that the performance was a significant improvement over the classical MSE-based method.

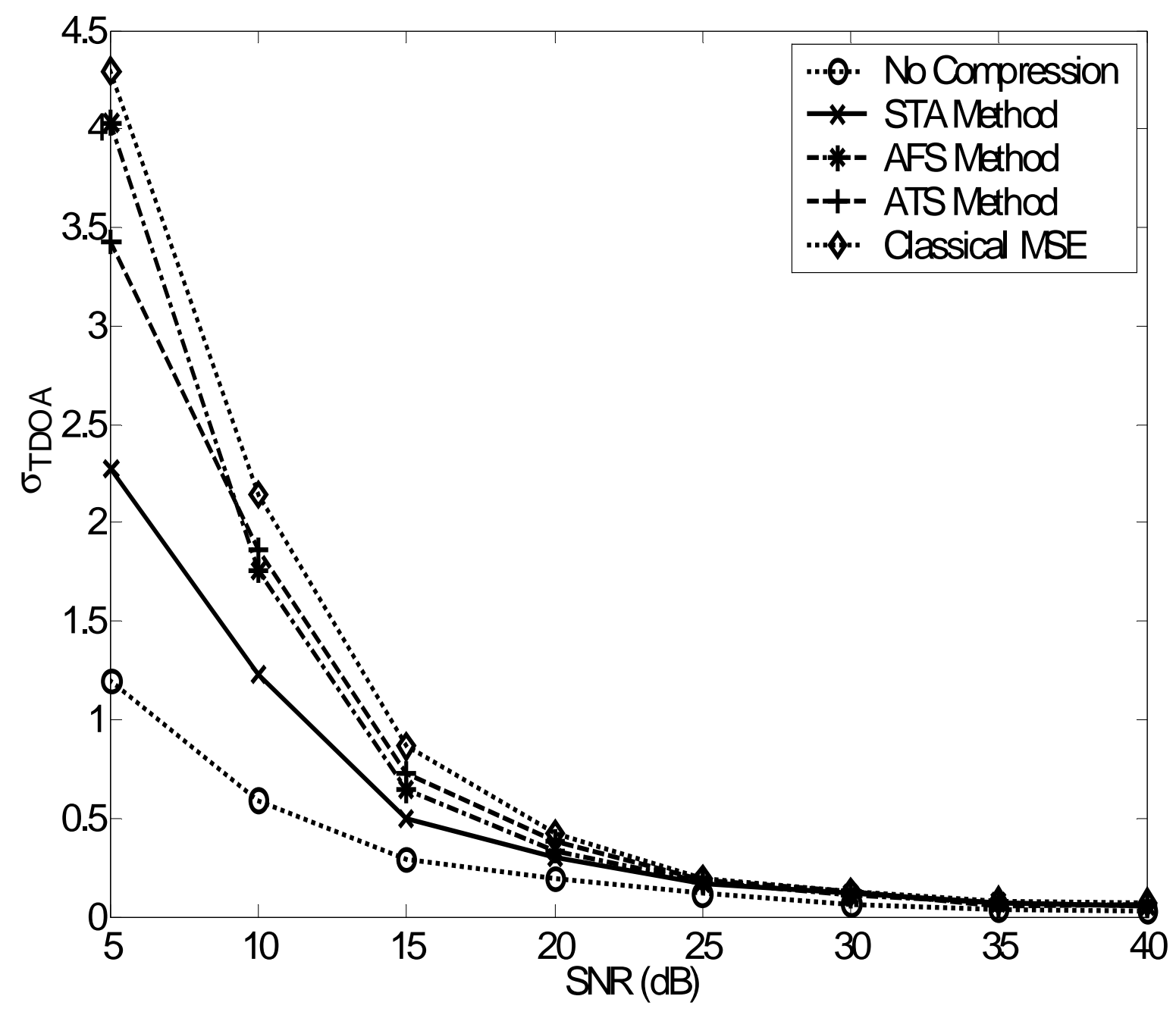

Figure 2: TDOA Accuracy versus SNR for several compression methods for a 2.5:1 compression ratio.

\section{REFERENCES}

1. P. C. Chestnut, "Emitter location accuracy using TDOA and differential doppler," IEEE Trans. Aero. and Electronic Systems, vol. AES-18, pp. 214-218, March 1982.

2. S. Stein, "Differential delay/doppler ML estimation with unknown signals," IEEE Trans. Sig. Proc., vol. 41, pp. 2717 2719, August 1993.

3. S. Stein, "Algorithms for ambiguity function processing," IEEE Trans. Acoustics, Speech, and Signal Processing, vol. ASSP-29, pp. 588 - 599, June 1981.

4. D. J. Matthiesen and G. D. Miller, "Data transfer minimization for coherent passive location systems," Report No. ESDTR-81-129, Air Force Project No. 4110, June 1981. 
5. G. Desjardins, “TDOA/FDOA technique for locating a transmitter," US Patent \#5,570,099 issued Oct. 29, 1996, held by Lockheed Martin.

6. M. L. Fowler, "Coarse quantization for data compression in coherent location systems," IEEE Trans. Aerospace and Electronic Systems, vol. 36, no. 4, pp. 1269 - 1278, Oct. 2000.

7. M. L. Fowler, "Data compression for TDOA/DD-based location system," US Patent \#5,991,454 issued Nov. 23, 1999, held by Lockheed Martin.

8. M. L. Fowler, "Data compression for emitter location systems," Conference on Information Sciences and Systems, Princeton University, March 15-17, 2000, pp. WA7b-14 - WA7b-19.

9. M. L. Fowler, "Decimation vs. quantization for data compression in TDOA systems," in Mathematics and Applications of Data/Image Coding, Compression, and Encryption III, Mark S. Schmalz, Editor, Proceedings of SPIE Vol. 4122, pp. 56 - 67, San Diego, CA, July 30 - August 4, 2000.

10. M. L. Fowler, "Exploiting RMS Time-Frequency Structure for Data Compression in Emitter Location Systems," National Aerospace and Electronics Conference, Dayton, OH, October 10-12, 2000, pp. 227 - 234.

11. M. L. Fowler, "Non-MSE Wavelet-Based Data Compression for Emitter Location," in Mathematics and Applications of Data/Image Coding, Compression, and Encryption IV, Mark S. Schmalz, Editor, Proceedings of SPIE, Vol. 4475, San Diego, CA, July 29 - August 3, 2001, pp. 13 - 22.

12. K. Sayood, Introduction to Data Compression, $2^{\text {nd }}$ Edition, Morgan Kaufmann, 2000.

13. M. Vetterli and J. Kovacevic, Wavelets and Subband Coding, Prentice-Hall, 1995.

14. L. R. Foulds, Combinatorial Optimization for Undergraduates, New York: Springer-Verlag, 1984. 